\title{
Python on the Landscape of Programming Tools for Design and Architectural Education
}

\author{
Python on the Landscape of Programming Tools for Design and Architectural Education
}

\author{
Alexandre Barrozo do Amaral Villares \\ FEC-Unicamp, Brazil \\ abav@lugaralgum.com \\ Daniel de Carvalho Moreira \\ FEC-Unicamp, Brazil \\ damore@fec.unicamp.br
}

\begin{abstract}
Currently most professional modeling and computer graphics software packages embed a scripting language. This is an early report on collecting data about software applications and coding tools geared towards the educational environment, preparing a listing for further evaluation and analysis of platforms. An increase in the adoption of Python as the embedded scripting syntax in many established tools can already be recognized, therefore the creation of educational materials on Python for design and architectural education merits further attention. Other insights on the educational potential of the available tools might be gained by advancing the data collection and evaluation work.
\end{abstract}

Keywords: Education; Design; Architecture; Programming; Python.

\section{Introduction}

Nowadays most $C A D$ and $3 D$ modeling software packages used by architects and designers embed a scripting language, and they have also been used as introductory tools to teach coding from early on. Mark Burry in Scripting Cultures: Architectural design and programming (2011), identifies different uses of computer programming, like productivity aids and exploratory code, as well as describes his teaching experience using scripting tools embedded in CAD software:

"[...] Once I had a handle on this coding caper, I could see then that I could attempt to transcend whatever limitations software might impose on me as a designer, guiding this electronic instrument with the same authority I applied to my pen and compass. [...] The two problems that had encouraged me to step outside my professional comfort zone of compliant passenger to become front-seat driver were stimulated by a need to rid myself of repetitive work [...]. Very quickly, however, I could see that a prime motivation for coding on top of software was to augment my design practice by allowing me to work in ways hitherto impractical, and so scripting became a medium of experimentation ahead of productivity gain. [...] Within a year, scripting had infiltrated my teaching, and in 1993 I instituted an elective course in which the participants had to come up with two pieces of code: a productivity tool (this was to appease my senior CAD teaching colleagues and satisfy the school's curriculum priorities) and a design experimentation script. [...]"(p.29)

Burry also compiled a list of programming tools used by several correspondents. It included textual scripting languages, like VBA, and tools with node diagrams, like Generative Components, embedded on mainstream computer graphics applications, like AutoCAD, as well as stand alone development environments, like Processing (Reas \& Fry, 2011).

Collecting and organising information about programming languages embedded in applications used by designers and architects, and also coding tools with educational aim, could be useful for teachers who need to choose suitable tools and supporting resources. Others researchers in the field of design and architectural education might engage in further investigations like comparing platforms (Celani \& Vaz, 2012) and analyzing trends.

Resilience Design is a contemporary aspect of design that might greatly benefit from model simulations, tackling design complexity and other exploratory strategies that are most effective through engagement in coding by design practitioners.

\section{Methods}

The records started to be collected in 2015 and the following selection criteria was adopted:

- Drawing or 3D modeling software that embeds a scripting language on the user interface or allows automation with a very limited number of steps between programming and code execution.

- Tools aimed at teaching programming in a visual or graphic context.

Software Development Kits (SDKs), usually provided by mainstream computer graphics software houses, mostly offering $\mathrm{C}$ or $\mathrm{C}++$ resources, were excluded. These tools are aimed at professional programmers who wish to create 
plugins, and are not immediately accessible to designers and architects, the main application end users.

The following fields are being recorded:

- $\quad$ Evaluation level: 'Yes' means the authors saw first hand the tool being used or used them themselves, 'partial' means some documentation of use was consulted at the time of entry, 'no' means only a description of the tool was available, possibly provided by a correspondent;

- $\quad$ OS: Describes if the tool will run on MacOS, Windows and/or GNU/Linux;

- License: 'FOSS' (Free/Libre and Open Source Software), or 'Proprietary', and in this last case, reference to, if available, any educational license, free or at reduced cost;

- Host/IDE: The main CAD, modelling, game application or Integrated Development Environment to be used. (i.e. SketchUp, Rhinoceros, Minecraft, or Processing IDE);

- Language/Library: Other software components or libraries that enable the programming interface. (i.e. Ruby, Grasshopper, ComputerCraftEdu, or P5*JS);

- Syntax: An attempt to group tools by language syntax features (i.e. Ruby, nodes, blocks, or JavaScript). 'Nodes' groups visual scripting tools like Generative Components, Grasshopper and Marionette). 'Blocks' groups programming interfaces similar to Scratch;

- Main Uses: A brief description of uses encountered;

- Official Site: Attempt to locate an online resource regarded as the official source of the tool;

- Recommended reference: Attempt to locate useful reference resources.

Many entries have not yet been evaluated, and the evaluation and categorization methods themselves require further work. It can be noted there is some overlap between categories due to the way entries have been recorded. The research would benefit from clearer criteria for listing platforms with several scripting and syntax options (at this point some are listed as separate entries, others grouped together).

\section{Results}

The preliminary results are presented in Table 1, also available as a CSV table published on GitHub inviting collaboration by means of corrections and additions: $<$ https://github.com/villares/Resources-for-teachingprogramming >

At the time of this writing 43 tools have been listed, of which 32 at least superficially investigated, of which at least $20 \%$ ( 7 of 32) have substantial educational aims.

A preliminary look at this landscape seems to indicate strong presence of Python as an embedded language or option in programming tools, about $40 \%$ (18 of 43 listed, 14 of 32 partially reviewed entries), followed by visual/node based tools (like GC, Dynamo and Grasshopper) about 15\% (5 of 32), Lua and BASIC-related (like GLD and VB.NET) about 15\% (5 of 32).

\section{Discussion}

There seems to be a trend of adoption of Python as an embedded scripting language. Python was added to Rhinoceros, arguably as replacement of RhinoScript, added to Vectorworks replacing VectorScript (a Pascal based language) and similarly Python was added to Maya, 3D Max and Cinema 4D.

On the Free/Libre and Open-Source category, Blender and FreeCAD have strong Python integration from the start. Rosetta, a CAD controlling extension used within DrRacket IDE, can now be accessed via Processing or Python as well (Caetano \& Leitão, 2016; Ramos \& Lleitão, 2014). Recently the Processing Foundation incorporated the Processing Python Mode as an official project (Parrish, 2016).

It can be noted that the Python programming language has grown in use at introductory courses in Computer Science and Engineering (Guo, 2014) and many other educational environments. According to Tollervey (2015), Python popularity in education might be explained from its origins on $A B C$, designed for teaching and aimed at nonprofessional programmers as well as on the open-source and extensible platform ecosystem "capable of simply and effectively addressing many different types of computational problems".

This leads to the preliminary conclusion that documenting Python use, and related software tools, as well as the creation of educational materials on Python for design and architectural education merit further work. It could be investigated, as further research, if knowledge of Python can be usefully transferred by students between platforms and tools.

Other insights on the evolution of the available scripting tools might be expected to follow from the accumulation of detailed data, it is mostly the intention of this work to provide a useful database for other researchers and specially teachers willing to explore further those tools. 
SIGraDi 2017, XXI Congreso de la Sociedad Ibero-americana de Gráfica Digital

22 -24 November, 2017 - Concepción, Chile.

Table 1: Data collected up to May 2017 (part 1 of 2)

\begin{tabular}{|c|c|c|c|c|c|c|c|}
\hline ID & Evaluation & os & License & Host/IDE & Language/Library & Syntax & Main uses \\
\hline 10 & yes & Windows, Mac OS & $\begin{array}{l}\text { Proprietary, educational } \\
\text { use at no cost }\end{array}$ & ArchiCAD & GDL & BASIC-like & Parametric objects, complex 3D geometry \\
\hline 20 & yes & $\begin{array}{l}\text { Windows, Mac OS. } \\
\text { Linux }\end{array}$ & FOSS & Arduino & CMiring & C-like & $\begin{array}{l}\text { Physical computing, home automation. } \\
\text { interactive art. }\end{array}$ \\
\hline 30 & yes & Windows & Proprietary, no cost licence & Bentley & $\begin{array}{l}\text { Generative } \\
\text { Components }\end{array}$ & nodes & $\begin{array}{l}\text { generative 3D geometry, complex 3D } \\
\text { geometry }\end{array}$ \\
\hline 40 & yes & $\begin{array}{l}\text { Windows, Mac OS. } \\
\text { Linux }\end{array}$ & FOSS & Blender & Python & Python & Plug-in tools, complex 3D geometry \\
\hline 50 & yes & Windows, Mac OS & FOSS & Cinder & $\mathrm{C}++$ & C-like & 2D graphics, generative art, dataviz \\
\hline 60 & yes & Mac OS & FOSS & Drawbot & Python & Python & 2D graphics, generative art, dataviz \\
\hline 70 & yes & $\begin{array}{l}\text { Windows, Mac OS, } \\
\text { Linux }\end{array}$ & FOSS & Processing & Processing Java Mode & Java & $\begin{array}{l}2 \mathrm{D} \text { and } 3 \mathrm{D} \text { graphics, intetactive art, } \\
\text { generative art, dataviz }\end{array}$ \\
\hline 71 & yes & $\begin{array}{l}\text { Windows, Mac OS, } \\
\text { Linux }\end{array}$ & FOSS & Processing & $\begin{array}{l}\text { Processing Python } \\
\text { Mode }\end{array}$ & Python & $\begin{array}{l}\text { 2D and 3D graphics, intetactive art, } \\
\text { generative art, dataviz }\end{array}$ \\
\hline 72 & yes & $\begin{array}{l}\text { Windows, Mac OS, } \\
\text { Linux }\end{array}$ & FOSS & $\begin{array}{l}\text { P5*JS (Processing } \\
\text { Foundation) }\end{array}$ & P5*JS & JavaScript & $\begin{array}{l}\text { 2D and } 3 \mathrm{D} \text { graphics, intetactive art, } \\
\text { generative art, dataviz }\end{array}$ \\
\hline 80 & yes & Windows & $\begin{array}{l}\text { Proprietary, educational } \\
\text { use at no cost or reduced } \\
\text { cost }\end{array}$ & $\begin{array}{l}\text { Revit, Vasari \& } \\
\text { Dynamo Studio }\end{array}$ & Dynamo & nodes. DesignScript & Generative geometry in 3D \\
\hline 90 & yes & Windows & $\begin{array}{l}\text { Proprietary, educational at } \\
\text { reduced cost }\end{array}$ & Rhinoceros & Grasshopper & nodes & Generative geometry in $3 D$ \\
\hline 91 & yes & Windows, Mac OS & $\begin{array}{l}\text { Proprietary, educational at } \\
\text { reduced cost }\end{array}$ & Rhinoceros & Python & Python & Plug-in tools, complex 3D geometry \\
\hline 92 & yes & Windows, Mac OS & $\begin{array}{l}\text { Proprietary, educational at } \\
\text { reduced cost }\end{array}$ & Rhinoceros & Rhinoscript & VBScript & Plug-in tools, complex 3D geometry \\
\hline 100 & yes & $\begin{array}{l}\text { Windows, Mac OS. } \\
\text { Linux }\end{array}$ & FOSS & Scratch & Scratch & blocks & Education, games \\
\hline 110 & yes & Windows, Mac OS & $\begin{array}{l}\text { Proprietary, educational } \\
\text { use at no cost or reduced } \\
\text { cost }\end{array}$ & SketchUp & Ruby & Ruby & Plug-in tools, complex 3D geometry \\
\hline 120 & yes & Windows. Mac OS & $\begin{array}{l}\text { Proprietary. educational } \\
\text { use at no cost }\end{array}$ & Vectorworks & Marionette & nodes & Generative geometry in 3D \\
\hline 121 & yes & Windows, Mac OS & $\begin{array}{l}\text { Proprietary, educational } \\
\text { use at no cost }\end{array}$ & Vectorworks & Vectorscript & Pascal & $\begin{array}{l}\text { Plug-in tools, parametric objects, complex } \\
\text { 3D geometry }\end{array}$ \\
\hline 122 & yes & Windows, Mac OS & $\begin{array}{l}\text { Proprietary, educational } \\
\text { use at no cost }\end{array}$ & Vectorworks & Python & Python & $\begin{array}{l}\text { Plug-in tools, parametric objects, complex } \\
\text { 3D geometry }\end{array}$ \\
\hline 130 & yes & online & $\cdot$ & $\begin{array}{l}\text { Trinket.io } \\
\text { (Browser based) }\end{array}$ & Python & $\begin{array}{l}\text { blocks, Python, } \\
\text { JavaScript }\end{array}$ & Education \\
\hline 140 & partial & Windows & Proprietary & ArcGIS & Python & Python & Plug-in tools, GIS, dataviz \\
\hline 150 & partial & Windows & $\begin{array}{l}\text { Proprietary, educational } \\
\text { use at no cost }\end{array}$ & Autocad & AutolLISP \& VisualLISP & Lisp-like & Plug-in tools, complex 2D and 3D geometry \\
\hline 151 & partial & Windows & $\begin{array}{l}\text { Proprietary, educational } \\
\text { use at no cost }\end{array}$ & Autocad & VB.NET & VB.NET & Plug-in tools, complex $2 \mathrm{D}$ and $3 \mathrm{D}$ geometry \\
\hline 160 & partial & $\begin{array}{l}\text { Windows, Mac OS, } \\
\text { Linux }\end{array}$ & FOSS & FreeCAD & Python & Python & Plug-in tools, complex 2D and 3D geometry \\
\hline 170 & partial & $\begin{array}{l}\text { Windows, Mac OS, } \\
\text { Linux }\end{array}$ & Proprietary & Minecraft & [various] & $\begin{array}{l}\text { blocks, Python, } \\
\text { JavaScript, Lua }\end{array}$ & Education, games \\
\hline 180 & partial & $\begin{array}{l}\text { Windows, Mac OS, } \\
\text { Linux }\end{array}$ & FOSS & NodeBox 3 & NodeBox & nodes, Python & 2D graphics, generative art, dataviz \\
\hline 181 & partial & $\begin{array}{l}\text { Windows, Mac OS, } \\
\text { Linux }\end{array}$ & FOSS & NodeBox for OpenGL & Python & Python & $2 \mathrm{D}$ graphics, generative art, dataviz \\
\hline 190 & partial & $\begin{array}{l}\text { Windows, Mac OS, } \\
\text { Linux }\end{array}$ & FOSS & OpenFrameworks & $\mathrm{C}++^{+}$ & C-like & 2D and 3D graphics, interactive art, dataviz \\
\hline 200 & partial & $\begin{array}{l}\text { Mac OS (aplicativo } \\
\text { ou módulo Python). } \\
\text { Windows e Linux } \\
\text { (módulo Python) }\end{array}$ & FOSS & PlotDevice & Python & Python & 2D graphics, generative art, dataviz \\
\hline 210 & partial & $\begin{array}{l}\text { Windows, Mac OS, } \\
\text { Linux }\end{array}$ & FOSS & QGIS & Python & Python & Plug-in tools, GIS, dataviz \\
\hline 81 & partial & Windows & $\begin{array}{l}\text { Proprietary. educational } \\
\text { use at no cost }\end{array}$ & Revit & $\begin{array}{l}\text { Revit Macros (VB.NET } \\
\text { or C\#) }\end{array}$ & VB.NET, C-like & $\begin{array}{l}\text { Plug-in tools, generative 3D geometry, } \\
\text { complex } 3 \mathrm{D} \text { geometry }\end{array}$ \\
\hline 220 & partial & online & - & $\begin{array}{l}\text { repl.it } \\
\text { (Browser based) }\end{array}$ & $\begin{array}{l}\text { Python } \\
\text { [and many others] }\end{array}$ & Python [and others] & Education \\
\hline 230 & partial & $\begin{array}{l}\text { Windows, Mac OS, } \\
\text { Linux }\end{array}$ & FOSS & DrRackett & Racket/Rosetta & Lisp-like, Python, Java & $\begin{array}{l}\text { Education, generative } 3 \mathrm{D} \text { geometry, } \\
\text { complex 3D geometry }\end{array}$ \\
\hline 82 & no & Windows & $\begin{array}{l}\text { Proprietary, educational } \\
\text { use at no cost }\end{array}$ & Revit, Vasari & RevitPythonShell & Python & Plug-in tools, complex 3D geometry \\
\hline 240 & no & $\begin{array}{l}\text { Windows, Mac OS, } \\
\text { Linux }\end{array}$ & & Blued & Java & Java & Education \\
\hline 250 & no & $\begin{array}{l}\text { Windows, Mac OS, } \\
\text { Linux }\end{array}$ & & Greenfoot & Java & Java & Education \\
\hline 260 & no & Windows & Proprietary & 3D Max & Python & Python & 3D \\
\hline 261 & no & Windows & Proprietary & 3D Max & MAXScript & MAXScript & 3D \\
\hline 270 & no & Windows, Mac OS & Proprietary & Cinema 4D & Python & Python & 3D \\
\hline 271 & no & Windows, Mac OS & Proprietary & Cinema 4D & Coffee & Coffee & 3D \\
\hline 280 & no & & Proprietary & Maya & Python & Python & 3D \\
\hline 290 & no & & & Unity & $\mathrm{C \#}$ & C-like & games, dataviz \\
\hline 300 & no & & & Unreal Engine & Blueprints & blocks & games, dataviz \\
\hline 310 & no & $\begin{array}{l}\text { Windows, Mac OS, } \\
\text { Linux }\end{array}$ & Proprietary & Stencyl & Stencyl & blocks, Java & Education, games \\
\hline
\end{tabular}


SIGraDi 2017, XXI Congreso de la Sociedad Ibero-americana de Gráfica Digital 22 -24 November, 2017 - Concepción, Chile.

Table 1: Data collected up to May 2017 (part 2 of 2)

\begin{tabular}{|c|c|c|}
\hline ID & Official site & Recommended Ref. \\
\hline 10 & gdl.graphisoft.com/ & GDL Cookbook \\
\hline 20 & arduine.ce & \\
\hline 30 & $\begin{array}{l}\text { bentley.com/en/products/product-line/modeling-and- } \\
\text { visualization-software/generativecomponents }\end{array}$ & \\
\hline 40 & blender.org/manual/advanced/scripting/introduction.html & \\
\hline 50 & libcinder.orgl & \\
\hline 60 & unw. drawbot.com/ & \\
\hline 70 & processing.org & [listed separately on the GitHub repository] \\
\hline 71 & py.processing.org & [listed separately on the GitHub repository] \\
\hline 72 & D5js.org & [listed separately on the Gittlub repository] \\
\hline 80 & dynamobim.org/ & \\
\hline 90 & grasshopper3d.com/ & \\
\hline 91 & wiki.mcneel.com/developer/python & \\
\hline 92 & wiki.mcneel.com/developer/rhinoscript & \\
\hline 100 & scratch.mit.edul & scratch.mit.edu \\
\hline 110 & wuw.sketchup.com/int//en/developerf & Livro Automatic SketchUp \\
\hline 120 & developer.vectorworks.net/index.php/Marionette & www.techlimits.com/index.php/vectorworks-2016-marionete \\
\hline 121 & developer.vectorworks, netfindex,php/VectorScript & $\begin{array}{l}\text { https://stackoverflow,com/questions/263774/where-can-i- } \\
\text { find-resources-on-vectorscript-programming-language }\end{array}$ \\
\hline 122 & developer.vectorworks, netindex, php/Python & \\
\hline 130 & trinketio & hourofpython.com \\
\hline 140 & resources.arcgis.com/en/communities/python/ & \\
\hline 150 & $\begin{array}{l}\text { help. autodesk.com/view/ACD/2016/ENU/?guid=GUID- } \\
\text { 265AADB3-FB89-4D34-AA9D-6ADF70FF7D4B }\end{array}$ & \\
\hline 151 & $\begin{array}{l}\text { http://help, autodesk,com/view/ACD/2016/ENU/?quid=GUID- } \\
\text { 2CD40631-D67B-4DFO-A2C4-606EgB613252 }\end{array}$ & \\
\hline 160 & www.freecadweb.org & $\begin{array}{l}\text { http://www.freecadweb.org/wiki/index.php? } \\
\text { title=FreeCAD Scripting Basics }\end{array}$ \\
\hline 170 & & $\begin{array}{l}\text { http://computercraftedu.com/ http://pi.minecraft.net/? } \\
\text { page id }=14\end{array}$ \\
\hline 180 & wuw.nodebox,net/nodel & \\
\hline 181 & wuw.cityinabottle.org/nodeboxl & \\
\hline 190 & openframeworks.ccl & \\
\hline 200 & plotdevice.io & http://plotdevice.io/extras/plotdevice-docs.zip \\
\hline 210 & $\begin{array}{l}\text { docs.qgis. } \\
\text { org/testing/en/docs/pyagis developer cookbook/intro.html }\end{array}$ & ggistutorials.com/en/docs/getting started_with_pyqgis.html \\
\hline 81 & & $\begin{array}{l}\text { https://knowledge.autodesk.com/support/revit- } \\
\text { products/leam- } \\
\text { explore/caas/CloudHelp/cloudhelp/2016/ENU/Revit- } \\
\text { Customize/files/GUID-4DFDA8CD-BOFD-492E-8EDE- } \\
\text { A28C29B1E316-htm.html }\end{array}$ \\
\hline 220 & replit & \\
\hline 230 & $\begin{array}{l}\text { http://web.jst.uth.ot/antonio.menezes. } \\
\text { leitao/Rosetta/tutorials/introduction. htm| }\end{array}$ & $\begin{array}{l}\text { htto://web.ist.utl,ptantonio.menezes, } \\
\text { leitao/Rosetta/tutorials/introduction.html }\end{array}$ \\
\hline 82 & $\begin{array}{l}\text { github.com/architecture-building- } \\
\text { systems/revitpythonshell/blob/master/README,md }\end{array}$ & \\
\hline 240 & nuw.bluej.ergl & btto:i/mww, bluej,org/objects-first/ \\
\hline 250 & unw.greenfoot.org/door & http://www.greenfoot.org/book/ \\
\hline 260 & $\begin{array}{l}\text { docs.autodesk.com/3DSMAX/16/ENU/3ds-Max-Python-API- } \\
\text { Documentation/index.html }\end{array}$ & \\
\hline \multicolumn{3}{|l|}{261} \\
\hline \multicolumn{3}{|l|}{270} \\
\hline \multicolumn{3}{|l|}{271} \\
\hline \multicolumn{3}{|l|}{280} \\
\hline 290 & & https://unity3d.com/leam/tutorials/topics/scripting \\
\hline 300 & & $\begin{array}{l}\text { httos://docs. unrealengine. } \\
\text { com/latest//NT/Engine/Blueprints/GettingStarted/index.html }\end{array}$ \\
\hline
\end{tabular}




\section{Acknowledgments}

The authors would like to thank the many correspondents who have contributed to this research over the last two years.

\section{References}

Burry, M. (2011). Scripting cultures: architectural design and programming. Chichester: Wiley.

Reas, C., \& Fry, B. (2011). Processing Architecture. Perspecta, 44, 153-202. https://doi.org/10.2307/41662956

Celani, G., \& Vaz, C. E. V. (2012). CAD Scripting and Visual Programming Languages for Implementing Computational Design Concepts: A Comparison from a Pedagogical Point of View. International Journal of Architectural Computing, 10(1), 121-137. https://doi.org/10.1260/1478-0771.10.1.121

Guo, P. (2014). Python is now the most popular introductory teaching language at top us universities. BLOG @ Communications of the ACM, July, 47. http://cacm.acm.org/blogs/blog-cacm/176450python-is-now-the-most-popular-introductory-teaching-languageat-top-us-universities/fulltext
Ramos, P. P., \& Leitão, A. M. (2014). Implementing Python for DrRacket. In M. J. V. Pereira, J. P. Leal, \& A. Simões (Eds.), 3rd Symposium on Languages, Applications and Technologies (Vol. 38, pp. 127-141). Dagstuhl, Germany: Schloss Dagstuhl-LeibnizZentrum fuer Informatik. https://doi.org/10.4230/OASIcs.SLATE.2014.127

Tollervey, N. (2015). Python in Education. Sebastopol, CA: O'Reilly Media. Retrieved from http://www.oreilly.com/programming/free/python-in-education.csp

Caetano, I., \& Leitão, A. M. (2016). Using Processing with Architectural 3D Modelling. Herneoja, Aulikki; Toni Österlund and Piia Markkanen (Eds.), Complexity \& Simplicity - Proceedings of the 34th ECAADe Conference - Volume 1, University of Oulu, Oulu, Finland, 22-26 August 2016, Pp. 405-412. Retrieved from http://papers.cumincad.org/cgibin/works/Show?ecaade2016_199

Parrish, A., Fry, B., \& Reas, C. (2016). Getting Started with Processing.py: Making Interactive Graphics with Processing's Python Mode (1 edition). San Francisco, CA: Maker Media, Inc. 